\title{
Evolution Operators for Linearly Polarized Two-Killing Cosmological Models
}

\author{
J. Fernando Barbero G.* and Daniel Gómez Vergel ${ }^{\dagger}$ \\ Instituto de Estructura de la Materia, CSIC, Serrano 123, 28006 Madrid, Spain \\ Eduardo J. S. Villaseñor ${ }^{\ddagger}$ \\ Grupo de Modelización y Simulación Numérica, Universidad Carlos III de Madrid, \\ Avda. de la Universidad 30, 28911 Leganés, Spain and \\ Instituto de Estructura de la Materia, CSIC, Serrano 123, 28006 Madrid, Spain
}

(Dated: March 13, 2006)

\begin{abstract}
We give a general procedure to obtain non perturbative evolution operators in closed form for quantized linearly polarized two Killing vector reductions of general relativity with a cosmological interpretation. We study the representation of these operators in Fock spaces and discuss in detail the conditions leading to unitary evolutions.

PACS numbers: 04.60.Ds, 04.60.Kz, 04.62.+v
\end{abstract}

\section{INTRODUCTION}

The study of symmetry reductions has been a fruitful way to gain valuable insights into the behavior of general relativity in its quantum regime. This is so because they provide tractable models where it is possible to make computations and obtain concrete predictions, at least in a qualitative way, about relevant features of a full blown theory of quantum gravity. Two Killing vector reductions are specially appealing because they have local degrees of freedom and (restricted) diffeomorphism invariance, two of the features of general relativity that lie at the heart of the difficulties encountered in its quantization. These reductions differ from each other in the spacetime topology and the action of the isometry group. When the Killing fields commute and are hypersurface orthogonal the models become specially simple and it is possible to solve them in closed form in a straightforward way. In some cases the reduced model describes the propagation of linearly polarized wave-like modes in a spacetime with non-compact spatial slices, the so called Einstein-Rosen waves [1]. Here the symmetry group is $\mathbb{R} \times U(1)$ and the spacetime is topologically $\mathbb{R}^{4}$. In other situations, when the symmetry group is $U(1) \times U(1)$, it provides cosmological models with initial singularities and an assortment of spatial topologies: There are non-compact examples such as the model introduced in [2] (referred to in the following as the Schmidt model) and compact ones such as the well known Gowdy $T^{3}$ model [3, 4], among many others. All these symmetric sectors of vacuum general relativity share the fact that the reduced phase space can be parametrized by a scalar field in $1+1$ dimensions and its canonically conjugate momentum (plus, eventually, some particle-like degrees of freedom). The main difference between them, as far as the quantization is concerned, is that the Hamiltonian for Einstein-Rosen waves [5] is time independent and has its origin in the surface terms needed to have a well-defined action principle whereas the Hamiltonian in the cosmological models is time dependent and is obtained through deparametrization $[6,7]$.

The quantization of linear Einstein-Rosen waves (free [5, 8, 9, 10] or coupled to matter [11]) is fairly well understood. The quantum unitary evolution operator can be obtained in closed form in a straightforward way and can be used for a number of purposes leading to physical applications such as the discussion of the existence of large quantum gravity effects [10] or the study of the microcausality of the system [12]. Models of the cosmological type, specially the Gowdy $T^{3}$ one, have been much harder to crack even though they received a lot of attention already in the seventies by Misner [13], Berger [14], and other authors. Although the first attempts to quantize the system were largely successful [15] and key technical insights were introduced already at these stage some features of the formalism were not completely satisfactory. In particular, the reliance on a Hilbert space built as a tensor product of infinite, one-particle Hilbert spaces is problematic since it is known that such a tensor product is not separable and the representation of the canonical commutation relations that it provides is reducible [16]. A renewed interest in the quantization of the Einstein-Rosen, Gowdy, and Schmidt models arose in the nineties $[5,6,7,9,10]$ when, instead of the Dirac approach previously used, the quantization of the system was done by gauge fixing. At the time it was thought that they could

\footnotetext{
*fbarbero@iem.cfmac.csic.es

$\dagger$ dgvergel@iem.cfmac.csic.es

¥ejsanche@math.uc3m.es
} 
provide suitable testing grounds for loop quantum gravity and address some general issues related to the quantization of general relativity. However the finding that there were problems with the unitary implementation of dynamics in the Gowdy $T^{3}$ model (and also in the Schmidt case) [17, 18] was somewhat of a shock and was perceived as a potential drawback to its use as a toy model for quantum gravity. The situation has recently improved after Corichi et. al. $[19,20]$ have shown the existence of unitary evolution for a field closely related to the scalar that usually encodes the local gravitational degrees of freedom.

The purpose of the present paper in this context is to obtain evolution operators explicitly in closed form written in terms of the basic objects, i.e. the field and momentum operators. This is done before choosing a specific Hilbert space representation for them. So, even if they are formal at this stage, they offer the possibility to explore different choices for the quantization of the system and, in particular, non-Fock representations (when available). Owing to this fact it is important to notice that our approach differs from the usual ones that make use of the Fock space -built from the solutions to the field equations- and a choice of complex structure to select the one-particle Hilbert space. As an application of our formalism we will discuss the unitary implementability of the time evolution of the system as done in [19] by changing the basic fields used to encode the physical degrees of freedom of the model. This provides an alternative point of view on this problem.

The outline of the paper is the following. After this introduction we review in section II the Hamiltonian formalism for the $U(1) \times U(1)$ symmetric models considered in the paper; specifically we will discuss their derivation from an action principle, gauge fixing, and deparametrization. After this we will devote section III to obtain formal unitary evolution operators $U\left(t, t_{0}\right)$, defined in terms of abstract field and momentum. This will be done in a unified way for a family of time dependent Hamiltonians that include the Schmidt and Gowdy models. Under the condition that the above mentioned formal operators can be made unitary by choosing a suitable representation for the field on a Hilbert space they can be shown to satisfy the evolution equation $i \hbar \partial_{t} \hat{U}\left(t, t_{0}\right)=\hat{H}(t) \hat{U}\left(t, t_{0}\right)$ where $\hat{H}(t)$ is the time dependent Hamiltonian of the system. The technical details of the construction -that relies upon known results concerning the quantization of the time-dependent harmonic oscillator- will be left to appendix A.

As an application of our scheme we discuss in section IV the problem of finding suitable Fock space representations for the field and its momentum that give rise to unitary evolution. At this stage the use of the auxiliary field considered in [14] and reintroduced in $[19,20]$ will play an important role that will be clarified within the present framework. We end the paper with our conclusions in section $\mathrm{V}$ and three appendices where we provide mathematical details on the obtention of the evolution operators and related topics.

\section{LINEARLY POLARIZED $U(1) \times U(1)$ MODELS: HAMILTONIAN FORMULATION}

The Gowdy and Schmidt models have been extensively used to study issues in quantum cosmology. In this context they are interesting because they share with the full theory of general relativity some of the features that renders its quantization highly non-trivial, in particular, they are genuine field theories with (some) diffeomorphism invariance. They have also been used to discuss physical issues such as the quantum behavior of the initial singularity in a non-homogeneous setting $[14,21]$.

\section{A. The Midi-Superspace}

We will start by reviewing here some relevant facts about the Gowdy and Schmidt models, in particular, the symmetry reduction process and the deparametrization needed to define a convenient time-dependent Hamiltonian. The spacetimes $\left(\mathcal{M},{ }^{(4)} g_{a b}\right)$ of the Schmidt and Gowdy $T^{3}$ models $[2,4]$ are vacuum solutions to the Einstein equations characterized by their isometry group, in this case $U(1) \times U(1)$. Once the group action is properly defined (smooth, effective, proper, and with no fixed points ${ }^{1}$ ) on the connected and oriented spatial sections $\Sigma^{3}$ of $\mathcal{M}=\mathbb{R} \times \Sigma^{3}$ the spatial topology is fixed to be of the form $\Sigma^{3}=\mathbb{X} \times \mathbb{S}^{1} \times \mathbb{S}^{1}$, where $\mathbb{X}=\mathbb{R}$ for Schmidt and $\mathbb{X}=\mathbb{S}^{1}$ for Gowdy $[22,23]$.

We will focus here on the linearly polarized case where the isometry group is generated by a pair of mutually orthogonal, commuting, spacelike, and globally defined hypersurface-orthogonal Killing vector fields $\left(\xi^{a}, \sigma^{a}\right)$. The symmetry of the system implies that the Lie derivatives of the metric vanish: $L_{\xi}^{(4)} g_{a b}=L_{\sigma^{(4)}} g_{a b}=0$.

With these assumptions it is possible to exactly solve the vacuum Einstein equation

$$
{ }^{(4)} R_{a b}=0,
$$

\footnotetext{
1 This condition rules out the $\mathbb{S}^{1} \times \mathbb{S}^{2}, \mathbb{S}^{3}$, and lens space topologies for the Gowdy model.
} 
where ${ }^{(4)} R_{a b}$ is the Ricci tensor associated with the Levi-Civita connection ${ }^{(4)} \nabla_{a}$ compatible with the 4 -metric ${ }^{(4)} g_{a b}$. Since the Killing fields are hypersurface orthogonal, the space of orbits $M=\mathbb{R} \times \mathbb{X} \times \mathbb{S}^{1}$ defined by one of them, say $\xi^{a}$, can be identified as an embedded hypersurface in $\mathcal{M}$ which is everywhere orthogonal to the (closed) orbits of $\xi^{a}$. The induced 3-metric ${ }^{(3)} g_{a b}$ on $M$ can be written in terms of $\xi_{a}:={ }^{(4)} g_{a b} \xi^{b}$ and $\lambda:={ }^{(4)} g_{a b} \xi^{a} \xi^{b}>0$ as

$$
{ }^{(3)} g_{a b}={ }^{(4)} g_{a b}-\lambda^{-1} \xi_{a} \xi_{b}
$$

Let ${ }^{(3)} R_{a b},{ }^{(3)} \nabla_{a}$, and ${ }^{(3)} \square$ be, respectively, the Ricci tensor, the metric connection, and the d'Alembert operator associated to ${ }^{(3)} g_{a b}$. The equation (1) is then equivalent to the system [24]

$$
\begin{aligned}
& { }^{(3)} \square \lambda=\frac{1}{2 \lambda}{ }^{(3)} g^{a b}\left({ }^{(3)} \nabla_{a} \lambda\right)\left({ }^{(3)} \nabla_{b} \lambda\right), \\
& { }^{(3)} R_{a b}=\frac{1}{2 \lambda}{ }^{(3)} \nabla_{a}{ }^{(3)} \nabla_{b} \lambda-\frac{1}{4 \lambda^{2}}\left({ }^{(3)} \nabla_{a} \lambda\right)\left({ }^{(3)} \nabla_{b} \lambda\right)
\end{aligned}
$$

The remaining Killing vector field $\sigma^{a}$ satisfies $L_{\sigma}^{(3)} g_{a b}=0, L_{\sigma} \lambda=0$.

It is well known that in order to further simplify the equations (3) we can use the conformal transformation

$$
g_{a b}:=\lambda^{(3)} g_{a b}
$$

that is well defined since $\xi^{a}$ never vanishes. Let $R_{a b}, \nabla_{a}$, and $\square$ be, respectively, the Ricci tensor, the metric connection and the d'Alembert operator associated to the new 3-metric $g_{a b}$. After the conformal rescaling (4), the system (3) is equivalent to

$$
\begin{aligned}
& \square \phi=0, \\
& R_{a b}=\left(\nabla_{a} \phi\right)\left(\nabla_{b} \phi\right),
\end{aligned}
$$

where we have defined the field $\sqrt{2} \phi:=\log \lambda$. Therefore, the symmetry reduced models considered here can be thought of as $(2+1)$-general relativity coupled to a massless scalar field $\phi$, with the additional symmetry defined by the Killing field $\sigma^{a}$. They can be derived from the $2+1$ Einstein-Hilbert action for gravity coupled to a massless scalar

$$
S[g, \phi]=\frac{1}{16 \pi G_{3}} \int_{M}|g|^{1 / 2}\left(R-g^{a b} \nabla_{a} \phi \nabla_{b} \phi\right)+\frac{1}{8 \pi G_{3}} \oint_{\partial M}|h|^{1 / 2} K,
$$

that we will use as the starting point for the Hamiltonian formalism. The last term must be introduced in the noncompact case in order to have a well defined variational principle and involves an integration over the 2-dimensional asymptotic boundary of $M$. Here $R$ is the scalar curvature of $g_{a b}, h$ and $K$ are, respectively, the determinant of the induced metric and the trace of the second fundamental form of the boundary $\partial M$, and $G_{3}$ is the Newton constant per unit length in the direction of the symmetry orbits.

In order to respect the symmetry in the Hamiltonian analysis of (6) it is important to notice that, owing to the hypersurface orthogonality condition, the metric can be decomposed as

$$
g_{a b}=h_{a b}+\tau^{-2} \sigma_{a} \sigma_{b}
$$

where $\sigma_{a}:=g_{a b} \sigma^{b}, \tau^{2}:=\sigma_{a} \sigma^{a}$ is the area density of the symmetry group orbits, and $h_{a b}$ denotes the induced metric of signature $(-+)$ on the 2-dimensional manifolds -topologically $\mathbb{R} \times \mathbb{X}$ - that are everywhere orthogonal to the closed orbits of $\sigma^{a}$. The symmetry present in this case implies that the gradient of $\tau$ is always timelike. We introduce now a foliation of $\mathbb{R} \times \mathbb{X}$ defined by spacelike level hypersurfaces of a suitable scalar function $t$. We also define a dynamical vector field $t^{a}$ such that $t^{a} \nabla_{a} t=1$. By using the unit, future-pointing, timelike, normal vector field to the foliation $n^{a}$ and the unit vector field $\hat{x}^{a}$ compatible with the chosen orientation and tangent to each slice (topologically $\mathbb{X}$ ) it is possible to write $t^{a}=N n^{a}+N^{x} \hat{x}^{a}$, where $N$ and $N^{x}$ are the lapse and shift functions. The 2-metric $h_{a b}$ can be written then as $h_{a b}=-n_{a} n_{b}+\hat{x}_{a} \hat{x}_{b}$, where $n_{a}$ and $\hat{x}_{a}$ are respectively $g_{a b} n^{b}$ and $g_{a b} \hat{x}^{b}$. We introduce the additional non-unit vector field $x^{a}=\mathrm{e}^{\gamma / 2} \hat{x}^{a}$, where $\gamma$ is an extra field, and impose the vanishing of the Lie brackets of the set of vector fields $\left(t^{a}, x^{a}, \sigma^{a}\right)$ :

$$
\begin{aligned}
& \partial_{\sigma} N=\partial_{\sigma} N^{x}=\partial_{\sigma} \gamma=0, \\
& {[\sigma, \hat{x}]^{a}=[\sigma, n]^{a}=0,} \\
& N \mathrm{e}^{\gamma / 2}[\hat{x}, n]^{a}=-n^{a} \partial_{x} N-\hat{x}^{a}\left(\partial_{x} N^{x}-\partial_{t} \mathrm{e}^{\gamma / 2}\right) .
\end{aligned}
$$


By doing this it is possible to construct a global coordinate system for $M:(t, x, \sigma) \in(0, \infty) \times \mathbb{X} \times \mathbb{S}^{1}$. In these coordinates

$$
g_{a b}=\left(-N^{2}+\left(N^{x}\right)^{2}\right) \nabla_{a} t \nabla_{b} t+2 \mathrm{e}^{\gamma / 2} N^{x} \nabla_{(a} t \nabla_{b)} x+\mathrm{e}^{\gamma} \nabla_{a} x \nabla_{b} x+\tau^{2} \nabla_{a} \sigma \nabla_{b} \sigma,
$$

where the scalar fields $N, N^{x}$, and $\gamma$ depend only on $t$ and $x$. Thus, the midi-superspace under consideration consists of five real-valued smooth functions $\left(N, N^{x}, \tau, \gamma, \phi\right)$ that depend only on $(t, x)$ and satisfy the Einstein-Klein-Gordon field equations (5) with the metric (9). In the Gowdy model, these functions are periodic in $x$. However, in the Schmidt model $x \in \mathbb{R}$ and we need to impose asymptotic conditions for the fields in the limits $x \rightarrow \pm \infty$. Here we will make use of the same conditions introduced in [7]

$$
\begin{array}{ll}
\gamma \rightarrow \gamma_{ \pm}(t)+O\left(x^{-1}\right), & N \rightarrow N_{ \pm}(t)+O\left(x^{-1}\right), \\
\tau \rightarrow \tau_{ \pm}(t)+O\left(x^{-1}\right), & N^{x} \rightarrow O\left(x^{-1}\right), \\
\phi \rightarrow O\left(x^{-1}\right) . &
\end{array}
$$

Due to these fall-off conditions for the fields the boundary integral in the action (6) vanishes in the non-compact case (it is trivially zero in the compact one).

Introducing the metric (9) in the action (6) it is straightforward to write it in the canonical form

$$
S[g, \phi]=\int_{t_{1}}^{t_{2}}\left(\int_{\mathbb{X}}\left(p_{\gamma} \dot{\gamma}+p_{\tau} \dot{\tau}+p_{\phi} \dot{\phi}\right) \mathrm{d} x-H\left[N, N^{x}\right]\right) \mathrm{d} t,
$$

where the dot denotes $\partial_{t}, p_{\gamma}, p_{\tau}$, and $p_{\phi}$ are the canonically conjugate momenta [with the fall-off conditions inherited from (10)], and $H\left[N, N^{x}\right]$ is the Hamiltonian that can be written as the sum of the first class constraints $C[N]$ and $C_{x}\left[N^{x}\right]$

$$
H\left[N, N^{x}\right]=C[N]+C_{x}\left[N^{x}\right]=\int_{\mathbb{X}} N C \mathrm{~d} x+\int_{\mathbb{X}} N^{x} C_{x} \mathrm{~d} x
$$

Here

$$
\begin{aligned}
& C:=\frac{1}{8 G_{3}} \mathrm{e}^{-\gamma / 2}\left(2 \tau^{\prime \prime}-\gamma^{\prime} \tau^{\prime}-\left(8 G_{3}\right)^{2} p_{\tau} p_{\gamma}+\frac{\left(8 G_{3}\right)^{2}}{4 \tau} p_{\phi}^{2}+\tau \phi^{\prime 2}\right) \\
& C_{x}:=\mathrm{e}^{-\gamma / 2}\left(p_{\tau} \tau^{\prime}-2 p_{\gamma}^{\prime}+p_{\gamma} \gamma^{\prime}+\phi^{\prime} p_{\phi}\right)
\end{aligned}
$$

and we have denoted $\partial_{x}$ by a prime. In the following we will choose units such that $8 G_{3}=1$ so that the previous constraints become

$$
\begin{aligned}
& C[N]=\int_{\mathbb{X}} N \mathrm{e}^{-\gamma / 2}\left(2 \tau^{\prime \prime}-\gamma^{\prime} \tau^{\prime}-p_{\tau} p_{\gamma}+\frac{1}{4 \tau} p_{\phi}^{2}+\tau \phi^{\prime 2}\right) \mathrm{d} x, \\
& C_{x}\left[N^{x}\right]=\int_{\mathbb{X}} N^{x} \mathrm{e}^{-\gamma / 2}\left(p_{\tau} \tau^{\prime}-2 p_{\gamma}^{\prime}+p_{\gamma} \gamma^{\prime}+\phi^{\prime} p_{\phi}\right) \mathrm{d} x,
\end{aligned}
$$

where $N$ and $N^{x}$ are arbitrary functions. As the Hamiltonian is zero -regardless of $\mathbb{X}$ - we have no dynamics so in order to proceed further we will deparameterize the theory and introduce a suitable phase space variable to play the role of time.

\section{B. Deparameterization and Reduced Phase Space}

Deparameterization has been discussed in general in many places (see, for example, [25]); for the system under consideration we closely follow $[6,7]$ where the reader is directed for details. The fact that symmetry forces the area density of the group orbits $\tau$ to have time-like gradient and the analogy of the present models with the Einstein-Rosen waves $^{2}$ suggests that we choose $\tau$ as our time variable by imposing

$$
\tau^{\prime}=0 \quad \text { and } \quad p_{\gamma}+1=0
$$

\footnotetext{
${ }^{2}$ For Einstein-Rosen waves the area density has a space-like gradient and the gauge fixing that simplifies the interpretation of the model leads to use it as a radial coordinate.
} 
The first condition implies that $\tau$ is constant on the spatial slices of the foliation whereas the second basically means that the same is true for $\dot{\tau}$. Note that the last condition is essentially equivalent to $p_{\gamma}^{\prime}=0$ because in the non-compact case we have imposed a fall-off behavior for $p_{\gamma}[7]$ forcing it to asymptotically approach -1 . These conditions imply that all the degrees of freedom but a single one -that will change from one slice to another and will become the time variable of our model- are eliminated. In the compact case one must use a gauge fixing of the type $p_{\gamma}+p=0$ for a constant $p$. This takes care of a global degree of freedom that must be considered (see [6]). As we are mostly interested in the field-theoretic aspects of quantization for these systems we will drop this here by using the condition $p_{\gamma}+1=0$ in both cases. At this point one can check that the gauge fixing conditions (13) are admissible by computing their Poisson brackets with the constraints [7]. One can also see that there is just one first class constraint, say $C\left[N=e^{\gamma / 2}\right]$, which cannot be solved after fixing the gauge. This is found by solving for the lapse and shift that give a zero Poisson bracket of the Hamiltonian with the gauge fixing conditions. In fact the choice $N=e^{\gamma / 2}$ will be such that $\left\{\tau, C\left[N=e^{\gamma / 2}\right]\right\}=1$ and, hence, we can identify $\tau$ with the time parameter $t$ of the system.

In these models there is a quadratic conserved momentum

$$
P=\int_{\mathbb{X}} p_{\phi} \phi^{\prime} \mathrm{d} x
$$

that is forced to be zero in the compact case. As a consequence of this the reduced phase space for the Gowdy model is non-linear.

In order to complete the characterization of the reduced phase space of the deparameterized theory we must solve the first class constraints together with the gauge-fixing conditions. When this is done we find that the action of the system can be completely expressed in terms of the field $\phi$ and its canonically conjugate momentum $p_{\phi}$ to give

$$
s\left[\phi, p_{\phi}\right]=\int_{t_{1}}^{t_{2}} \int_{\mathbb{X}}\left[p_{\phi} \dot{\phi}-\left(\frac{p_{\phi}^{2}}{4 t}+t \phi^{\prime 2}\right)\right] \mathrm{d} x \mathrm{~d} t .
$$

The field equations are equivalent to the Hamilton equations derived from the time-dependent Hamiltonian

$$
H(t)=\int_{\mathbb{X}}\left(\frac{p_{\phi}^{2}}{4 t}+t \phi^{\prime 2}\right) \mathrm{d} x
$$

Once these equations are solved - and the constraint has been taken into account for Gowdy- the full four dimensional metric can be built from their solutions by following in reverse the reduction process for the metric and using the fact that the $2+1$ dimensional metric (9), defined in $(0, \infty) \times \mathbb{X} \times \mathbb{S}^{1}$, becomes

$$
g_{a b}=\mathrm{e}^{\gamma}\left(-\nabla_{a} t \nabla_{b} t+\nabla_{a} x \nabla_{b} x\right)+t^{2} \nabla_{a} \sigma \nabla_{b} \sigma
$$

where $\gamma^{\prime}=p_{\phi} \phi^{\prime}$. This metric displays a curvature singularity at $t=0$. Finally it is important to remark [6, 7] that the reduced action (15) corresponds, precisely, to that of a massless scalar field (independent of the $\sigma$ coordinate) on a fixed background spacetime with a metric given by

$$
\stackrel{\circ}{g}_{a b}=-\nabla_{a} t \nabla_{b} t+\nabla_{a} x \nabla_{b} x+t^{2} \nabla_{a} \sigma \nabla_{b} \sigma .
$$

This metric is defined on a 3 -manifold $(0, \infty) \times \mathbb{X} \times \mathbb{S}^{1}$ and shows, again, the singular behavior of the model at $t=0$.

\section{EVOLUTION OPERATORS}

This section is devoted to study the quantization of quadratic, time dependent, field Hamiltonians such as (16). The main difficulty to obtain evolution operators in closed form is due to their explicit time dependence that precludes us from writing them as exponentials of the Hamiltonian. A first point to notice is that some of the functions of time that appear in (16) -say the one in the $p_{\phi}^{2}$ term- can be eliminated by a simple redefinition of the time variable. Let us consider, then, the problem of quantizing a system with a time dependent Hamiltonian of the general form

$$
H(t)=\int_{\mathbb{X}}\left(\frac{1}{4} \pi^{2}(x)+\Lambda(t) \pi(x) \varphi(x)+\omega^{2}(t) \varphi^{\prime 2}(x)\right) \mathrm{d} x .
$$

Here $\varphi(x)$ and $\pi(x)$ are a field and its canonically conjugate momentum $[\{\varphi(x), \pi(y)\}=\delta(x, y)]$ defined on $\mathbb{X}$, that may be either the real line $\mathbb{R}$ (Schmidt) or the circle $\mathbb{S}^{1}$ (Gowdy). The prime denotes the $x$-derivative. The time functions $\Lambda(t)$ and $\omega(t)$ will be determined by the particular model considered. We allow for cross terms -absent in 
(16)- involving fields and momenta as they will appear in the discussion of the unitarity problem. We diagonalize the Hamiltonian (18) by writing ${ }^{3}$

$$
\begin{aligned}
\varphi(x) & =\frac{1}{\sqrt{2 \pi}} \int_{\tilde{\mathbb{X}}} \cos \left(k x-\frac{\pi}{4}\right) \varphi(k) \mathrm{d} \mu(k), \\
\pi(x) & =\sqrt{\frac{2}{\pi}} \int_{\tilde{\mathbb{X}}} \cos \left(k x-\frac{\pi}{4}\right) \pi(k) \mathrm{d} \mu(k),
\end{aligned}
$$

where $\varphi(k)$ and $\pi(k)$ are also canonically conjugate $[\{\varphi(k), \pi(q)\}=\delta(k, q)$ with a Dirac or Kronecker delta]. Here $\tilde{\mathbb{X}}$ denotes the real line for $\mathbb{X}=\mathbb{R}$ and the integers $\mathbb{Z}$ when $\mathbb{X}=\mathbb{S}^{1}$. The measure $\mathrm{d} \mu(k)$ simply refers to the fact that the previous integral either extends to $\mathbb{R}$ or becomes a sum over the integers respectively. In terms of them we have

$$
H(t)=\frac{1}{2} \int_{\tilde{\mathbb{X}}}\left(\pi^{2}(k)+2 \Lambda(t) \pi(k) \varphi(k)+k^{2} \omega^{2}(t) \varphi^{2}(k)\right) \mathrm{d} \mu(k) .
$$

This somewhat unusual diagonalization has the advantage of decoupling the different modes right from the start and avoids the problems encountered in [6] with the non-diagonal form of the Hamiltonian in terms of creation and annihilation operators. As we can see this is a sum of time dependent uncoupled Hamiltonians that are closely related to the harmonic oscillator with a time dependent frequency (even when the $\Lambda(t) \pi(k) \varphi(k)$ term is present). In fact, denoting $\partial_{t}$ by a dot, the Hamilton equations derived from (20) lead to

$$
\ddot{\varphi}(k, t)+\Omega^{2}(k, t) \varphi(k, t)=0
$$

with

$$
\Omega^{2}(k, t):=k^{2} \omega^{2}(t)-\Lambda^{2}(t)-\dot{\Lambda}(t),
$$

that we will suppose to be positive in the following.

To quantize the model we promote the field and momentum to formal algebraic objects $\hat{\varphi}(k)$ and $\hat{\pi}(k)$ satisfying the canonical commutation relations $[\hat{\varphi}(k), \hat{\pi}(q)]=i \hbar \delta(k, q)$ and symmetrize the cross term in (20). In the main body of the paper we take $\hbar=1$. This way we obtain

$$
\hat{H}(t)=\frac{1}{2} \int_{\tilde{\mathbb{X}}}\left(\hat{\pi}^{2}(k)+\Lambda(t)[\hat{\pi}(k) \hat{\varphi}(k)+\hat{\varphi}(k) \hat{\pi}(k)]+k^{2} \omega^{2}(t) \hat{\varphi}^{2}(k)\right) \mathrm{d} \mu(k) .
$$

Explicit solutions for the evolution operator for problems similar to this have been known for a number of years (see, for example, $[26,27]$ ). Using them (see appendix A) we can write down the formal quantum evolution operator $\hat{U}\left(t, t_{0}\right)$ as the product

$$
\hat{U}\left(t, t_{0}\right)=\hat{\mathcal{D}}\left(t, t_{0}\right) \hat{\mathcal{S}}\left(t, t_{0}\right) \hat{\mathcal{R}}\left(t, t_{0}\right)
$$

with

$$
\begin{aligned}
& \hat{\mathcal{D}}\left(t, t_{0}\right):=\exp \left(-\frac{i}{2} \int_{\tilde{\mathbb{X}}}\left[\frac{\dot{\rho}\left(k, t_{0}\right)}{\rho\left(k, t_{0}\right)}-\frac{\dot{\rho}(k, t)}{\rho(k, t)}+\Lambda(t)-\Lambda\left(t_{0}\right)\right] \hat{\varphi}^{2}(k) \mathrm{d} \mu(k)\right) \\
& \hat{\mathcal{S}}\left(t, t_{0}\right):=\exp \left(\frac { i } { 2 } \int _ { \tilde { \mathbb { X } } } [ \operatorname { l o g } \frac { \rho ( k , t _ { 0 } ) } { \rho ( k , t ) } ] \left\{\hat{\varphi}(k)\left[\hat{\pi}(k)-\left(\frac{\dot{\rho}\left(k, t_{0}\right)}{\rho\left(k, t_{0}\right)}-\Lambda\left(t_{0}\right)\right) \hat{\varphi}(k)\right]\right.\right. \\
&\left.\left.+\left[\hat{\pi}(k)-\left(\frac{\dot{\rho}\left(k, t_{0}\right)}{\rho\left(k, t_{0}\right)}-\Lambda\left(t_{0}\right)\right) \hat{\varphi}(k)\right] \hat{\varphi}(k)\right\} \mathrm{d} \mu(k)\right), \\
& \hat{\mathcal{R}}\left(t, t_{0}\right):=\exp \left(-\frac{i}{2} \int_{\tilde{\mathbb{X}}}\left[\int_{t_{0}}^{t} \frac{d \tau}{\rho^{2}(k, \tau)}\right]\left\{\frac{\hat{\varphi}^{2}(k)}{\rho^{2}\left(k, t_{0}\right)}+\rho^{2}\left(k, t_{0}\right)\left[\hat{\pi}(k)-\left(\frac{\dot{\rho}\left(k, t_{0}\right)}{\rho\left(k, t_{0}\right)}-\Lambda\left(t_{0}\right)\right) \hat{\varphi}(k)\right]^{2}\right\} \mathrm{d} \mu(k)\right) .
\end{aligned}
$$

\footnotetext{
${ }^{3}$ We purposely use the same letter to represent a field and its transform.
} 
Here $\rho(k, t)$ is any solution to the Ermakov-Pinney equation ${ }^{4}$

$$
\ddot{\rho}(k, t)+\Omega^{2}(k, t) \rho(k, t)=\rho^{-3}(k, t)
$$

with $\Omega^{2}(k, t)$ defined in (21). It can be shown (see appendix B) that $\rho(k, t)$ never vanishes and $\hat{U}\left(t, t_{0}\right)$ is independent of the choice of this solution. It must be said that the previous way to factorize $\hat{U}\left(t, t_{0}\right)$ is by no means unique although this form is specially adapted to the discussion of its unitarity in the next section.

If we give a representation for the fields $\hat{\varphi}(k)$ and $\hat{\pi}(k)$ satisfying $[\hat{\varphi}(k), \hat{\pi}(q)]=i \delta(k, q)$ and such that the exponents (multiplied by $i)$ in $(23,24,25)$ are self-adjoint, it is straightforward to check that $\hat{U}\left(t_{0}, t_{0}\right)=\mathbb{I}, \hat{U}\left(t, t_{0}\right)^{-1}=\hat{U}\left(t, t_{0}\right)^{\dagger}$, $\hat{U}\left(t_{2}, t_{0}\right)=\hat{U}\left(t_{2}, t_{1}\right) \hat{U}\left(t_{1}, t_{0}\right)$, and $i \partial_{t} \hat{U}\left(t, t_{0}\right)=\hat{H}(t) \hat{U}\left(t, t_{0}\right)$ where $\hat{H}(t)$ is the quantum Hamiltonian $(22)$, i.e. $\hat{U}\left(t, t_{0}\right)$ is the quantum evolution operator of the system in this representation. The evolution of the field and momentum operators in the Heisenberg picture can then be computed in a straightforward way and shown to satisfy the classical equations of motion as expected (see appendix A).

\section{FOCK REPRESENTATIONS AND UNITARY IMPLEMENTABILITY OF TIME EVOLUTION}

\section{A. General Considerations}

As a first application of the previously derived formula we will discuss here the problem of finding Fock space representations for the field and momenta leading to unitary evolution operators. This is important because of the known obstruction to the implementability of time evolution for this model in some of the descriptions considered in the past. Our results in this regard strongly support the satisfactory resolution of this issue that appears in [19].

Let us suppose that we take a Fock space $\mathcal{F}$ and write (overbars denote complex conjugation)

$$
\hat{\varphi}(k)=f(k) \hat{a}_{k}+\bar{f}(k) \hat{a}_{k}^{\dagger}, \quad \hat{\pi}(k)=g(k) \hat{a}_{k}+\bar{g}(k) \hat{a}_{k}^{\dagger}
$$

in terms of the annihilation and creation operators $\hat{a}_{k}$ and $\hat{a}_{k}^{\dagger}$ with $\left[\hat{a}_{k}, \hat{a}_{q}^{\dagger}\right]=\delta(k, q)$. The functions $f$ and $g$ must satisfy

$$
f(k) \bar{g}(k)-\bar{f}(k) g(k)=i,
$$

[so that $[\hat{\varphi}(k), \hat{\pi}(q)]=i \delta(k, q)]$ but are otherwise arbitrary at this stage. We want to find $f$ and $g$ such that $\hat{\mathcal{D}}\left(t, t_{0}\right)$, $\hat{\mathcal{S}}\left(t, t_{0}\right), \hat{\mathcal{R}}\left(t, t_{0}\right)$-with normal ordered exponents to prevent the appearance of infinite phases- are unitary and differentiable in $t$. An efficient procedure to discuss this issue has been put forward by Torre in [17] by using the theory of unitary implementation of canonical transformations. Let us consider a general quantum operator of the following type

$$
\exp \left(i \int_{\tilde{\mathbb{X}}}\left[\lambda_{1}(k): \hat{\varphi}^{2}(k):+\lambda_{2}(k): \hat{\pi}^{2}(k):+\lambda_{3}(k): \hat{\varphi}(k) \hat{\pi}(k)+\hat{\pi}(k) \hat{\varphi}(k):\right] \mathrm{d} \mu(k)\right),
$$

where $\lambda_{1,2,3}$ are real functions of $k$. Notice that $\hat{\mathcal{D}}\left(t, t_{0}\right), \hat{\mathcal{S}}\left(t, t_{0}\right)$, and $\hat{\mathcal{R}}\left(t, t_{0}\right)$ are particular cases of it with a parametric dependence on $t$ and $t_{0}$. Introducing (27) in (29), the operator can be written as

$$
\exp \left(i \int_{\tilde{\mathbb{X}}}\left[\chi_{1}(k) \hat{a}_{k} \hat{a}_{k}+\bar{\chi}_{1}(k) \hat{a}_{k}^{\dagger} \hat{a}_{k}^{\dagger}+2 \chi_{2}(k) \hat{a}_{k}^{\dagger} \hat{a}_{k}\right] \mathrm{d} \mu(k)\right),
$$

where $\chi_{1}:=\lambda_{1} f^{2}+\lambda_{2} g^{2}+2 \lambda_{3} f g$, and $\chi_{2}:=\lambda_{1}|f|^{2}+\lambda_{2}|g|^{2}+\lambda_{3}(f \bar{g}+\bar{f} g)$ is a real function. We want to know now if the exponent (times $i$ ) defines a self-adjoint operator. For fixed values of $t$ and $t_{0}$ this is done by studying the auxiliary dynamics -in a fictitious time parameter $s$ - defined by the exponent taken as a classical Hamiltonian

$$
F=\int_{\tilde{\mathbb{X}}}\left[\chi_{1}(k) a_{k} a_{k}+\bar{\chi}_{1}(k) \bar{a}_{k} \bar{a}_{k}+2 \chi_{2}(k) \bar{a}_{k} a_{k}\right] \mathrm{d} \mu(k),
$$

\footnotetext{
${ }^{4}$ The Ermakov-Pinney equation is of central importance in the classical and quantum treatment of the harmonic oscillator with timedependent frequency. It has been used, for example, in some field theoretic cosmological problems (see for example [28, 29]) and minisuperspace models $[30,31]$. In this paper we use it in the context of the Fock quantization of time dependent field theories.
} 
and using the results on quantum implementability that appear in [17]. The modes $a_{k}$ and $\bar{a}_{k}$ in (31) are defined by the classical field and momentum $\varphi(k)=f(k) a_{k}+\bar{f}(k) \bar{a}_{k}, \pi(k)=g(k) a_{k}+\bar{g}(k) \bar{a}_{k}$ and satisfy $\left\{a_{k}, \bar{a}_{q}\right\}=-i \delta(k, q)$. In practice it is convenient to consider the evolution equations

$$
\frac{\mathrm{d} a_{k}}{\mathrm{~d} s}=\left\{a_{k}, F\right\}=-2 i\left(\chi_{2}(k) a_{k}+\bar{\chi}_{1}(k) \bar{a}_{k}\right), \quad \frac{\mathrm{d} \bar{a}_{k}}{\mathrm{~d} s}=\left\{\bar{a}_{k}, F\right\}=2 i\left(\chi_{2}(k) \bar{a}_{k}+\chi_{1}(k) a_{k}\right)
$$

that are equivalent to the second order equations

$$
\frac{\mathrm{d}^{2} a_{k}}{\mathrm{~d} s^{2}}=4\left(\left|\chi_{1}(k)\right|^{2}-\chi_{2}^{2}(k)\right) a_{k}=4\left(\lambda_{3}^{2}(k)-\lambda_{1}(k) \lambda_{2}(k)\right) a_{k} .
$$

These are linear equations so their solutions have a linear dependence on the initial conditions $a_{k}\left(s_{0}\right)$ and $\mathrm{d} a_{k} / \mathrm{d} s\left(s_{0}\right)=$ $-2 i\left(\chi_{2}(k) a_{k}\left(s_{0}\right)+\bar{\chi}_{1}(k) \bar{a}_{k}\left(s_{0}\right)\right)$ and, hence, on $a_{k}\left(s_{0}\right)$ and $\bar{a}_{k}\left(s_{0}\right)$. In order to guarantee unitary implementability it suffices to show that the integral over $\tilde{\mathbb{X}}$ of the modulus squared of the coefficient of $\bar{a}_{k}\left(s_{0}\right)$ that appears in the solution of (32) is convergent. Finally, in order to verify the strong continuity of the transformation in the auxiliary parameter $s$ we have to check that the following limit

$$
\lim _{s \rightarrow s_{0}} \int_{\tilde{\mathbb{X}}}\left|a_{k}(s)-a_{k}\left(s_{0}\right)\right|^{2} \mathrm{~d} \mu(k)=0
$$

holds for the solution $a_{k}(s)$ of (32) with square summable initial data $a_{k}\left(s_{0}\right)$. We will obtain now general conditions that guarantee that the previously defined operators $\hat{\mathcal{D}}\left(t, t_{0}\right), \hat{\mathcal{S}}\left(t, t_{0}\right)$, and $\hat{\mathcal{R}}\left(t, t_{0}\right)(23)-(25)$ are unitary:

- $\hat{\mathcal{D}}\left(t, t_{0}\right)$ is a quantum operator of the form $(29)$ with $\lambda_{2,3}=0$ and $\lambda_{1} \neq 0$ given in terms of the solution to the Ermakov-Pinney equation (26) by

$$
\lambda_{1}(k)=\frac{1}{2}\left(\frac{\dot{\rho}(k, t)}{\rho(k, t)}-\Lambda(t)\right)-\frac{1}{2}\left(\frac{\dot{\rho}\left(k, t_{0}\right)}{\rho\left(k, t_{0}\right)}-\Lambda\left(t_{0}\right)\right) .
$$

In this case the solution to (32) is given by

$$
a_{k}(s)=\left[1-2 i\left(s-s_{0}\right) \chi_{2}(k)\right] a_{k}\left(s_{0}\right)-2 i\left(s-s_{0}\right) \bar{\chi}_{1}(k) \bar{a}_{k}\left(s_{0}\right)
$$

with $\chi_{1}=\lambda_{1} f^{2}$ and $\chi_{2}=\lambda_{1}|f|^{2}$. Then, the unitarity condition is

$$
\int_{\tilde{\mathbb{X}}} \lambda_{1}^{2}(k)|f(k)|^{4} \mathrm{~d} \mu(k)<\infty
$$

and the strong continuity condition (33) becomes

$$
0=\left.\left.\lim _{s \rightarrow s_{0}}\left(s-s_{0}\right)^{2} \int_{\tilde{\mathbb{X}}} \lambda_{1}^{2}(k)\left|\bar{f}^{2}(k) \bar{a}_{k}\left(s_{0}\right)+\right| f(k)\right|^{2} a_{k}\left(s_{0}\right)\right|^{2} \mathrm{~d} \mu(k) .
$$

This is trivially satisfied whenever the last integral is well defined.

- $\hat{\mathcal{S}}\left(t, t_{0}\right)$ is a quantum operator of the form $(29)$ with $\lambda_{2}=0, \lambda_{1,3} \neq 0$ :

$$
\begin{aligned}
& \lambda_{1}(k)=-\left(\frac{\dot{\rho}\left(k, t_{0}\right)}{\rho\left(k, t_{0}\right)}-\Lambda\left(t_{0}\right)\right) \log \frac{\rho\left(k, t_{0}\right)}{\rho(k, t)}, \\
& \lambda_{3}(k)=\frac{1}{2} \log \frac{\rho\left(k, t_{0}\right)}{\rho(k, t)} .
\end{aligned}
$$

The solution to $(32)$ is now

$$
\begin{aligned}
a_{k}(s) & =\left\{\cosh \left[2 \lambda_{3}(k)\left(s-s_{0}\right)\right]-i \chi_{2}(k) \lambda_{3}^{-1}(k) \sinh \left[2 \lambda_{3}(k)\left(s-s_{0}\right)\right]\right\} a_{k}\left(s_{0}\right) \\
& -i \bar{\chi}_{1}(k) \lambda_{3}^{-1}(k) \sinh \left[2 \lambda_{3}(k)\left(s-s_{0}\right)\right] \bar{a}_{k}\left(s_{0}\right),
\end{aligned}
$$

where $\chi_{1}=\lambda_{1} f^{2}+2 \lambda_{3} f g$, and $\chi_{2}=\lambda_{1}|f|^{2}+\lambda_{3}(f \bar{g}+\bar{f} g)$. 
The unitarity condition in this case is

$$
\int_{\tilde{\mathbb{X}}}|f(k)|^{2}\left|2 g(k)+\lambda_{1}(k) f(k) \lambda_{3}^{-1}(k)\right|^{2} \sinh ^{2}\left[2 \lambda_{3}(k)\left(s-s_{0}\right)\right] \mathrm{d} \mu(k)<\infty .
$$

It is important to point out here that the condition (28) implies that $|f(k)|^{2}|g(k)|^{2} \geq 1 / 4$ and, hence, the convergence of (36) requires an appropriate fall-off of $\left|1+\lambda_{1} f /\left(2 \lambda_{3} g\right)\right|^{2} \sinh ^{2}\left[2 \lambda_{3}\left(s-s_{0}\right)\right]$. The strong continuity condition is now

$$
\begin{gathered}
0=\lim _{s \rightarrow s_{0}} \int_{\tilde{\mathbb{X}}} \mid\left\{\cosh \left[2 \lambda_{3}(k)\left(s-s_{0}\right)\right]-i\left[f(k) \bar{g}(k)+\bar{f}(k) g(k)+\lambda_{1}(k) \lambda_{3}^{-1}(k)|f(k)|^{2}\right] \sinh \left[2 \lambda_{3}(k)\left(s-s_{0}\right)\right]-1\right\} a_{k}\left(s_{0}\right) \\
-\left.i\left[\lambda_{1}(k) \lambda_{3}^{-1}(k) \bar{f}^{2}(k)+2 \bar{f}(k) \bar{g}(k)\right] \sinh \left[2 \lambda_{3}(k)\left(s-s_{0}\right)\right] \bar{a}_{k}\left(s_{0}\right)\right|^{2} \mathrm{~d} \mu(k) .
\end{gathered}
$$

- Finally $\hat{\mathcal{R}}\left(t, t_{0}\right)$ is a quantum operator of the form (29) with $\lambda_{1,2,3} \neq 0$ given by

$$
\begin{aligned}
\lambda_{1}(k) & =-\frac{1}{2}\left[\frac{1}{\rho^{2}\left(k, t_{0}\right)}+\rho^{2}\left(k, t_{0}\right)\left(\frac{\dot{\rho}\left(k, t_{0}\right)}{\rho\left(k, t_{0}\right)}-\Lambda\left(t_{0}\right)\right)^{2}\right] \int_{t_{0}}^{t} \frac{d \tau}{\rho^{2}(k, \tau)}, \\
\lambda_{2}(k) & =-\frac{\rho^{2}\left(k, t_{0}\right)}{2} \int_{t_{0}}^{t} \frac{d \tau}{\rho^{2}(k, \tau)}, \\
\lambda_{3}(k) & =\frac{\rho^{2}\left(k, t_{0}\right)}{2}\left(\frac{\dot{\rho}\left(k, t_{0}\right)}{\rho\left(k, t_{0}\right)}-\Lambda\left(t_{0}\right)\right) \int_{t_{0}}^{t} \frac{d \tau}{\rho^{2}(k, \tau)}, \\
\text { with } \quad \lambda^{2}(k) & :=\lambda_{1}(k) \lambda_{2}(k)-\lambda_{3}^{2}(k)=\frac{1}{4}\left(\int_{t_{0}}^{t} \frac{d \tau}{\rho^{2}(k, \tau)}\right)^{2}>0 .
\end{aligned}
$$

The solution to (32) is given by

$$
\begin{aligned}
a_{k}(s) & =\left\{\cos \left[2 \lambda(k)\left(s-s_{0}\right)\right]-i \chi_{2}(k) \lambda^{-1}(k) \sin \left[2 \lambda(k)\left(s-s_{0}\right)\right]\right\} a_{k}\left(s_{0}\right) \\
& -i \bar{\chi}_{1}(k) \lambda^{-1}(k) \sin \left[2 \lambda(k)\left(s-s_{0}\right)\right] \bar{a}_{k}\left(s_{0}\right),
\end{aligned}
$$

with $\chi_{1}=\lambda_{1} f^{2}+\lambda_{2} g^{2}+2 \lambda_{3} f g$, and $\chi_{2}=\lambda_{1}|f|^{2}+\lambda_{2}|g|^{2}+\lambda_{3}(f \bar{g}+\bar{f} g)$.

Then, the unitarity condition is

$$
\int_{\tilde{\mathbb{X}}}\left|\lambda_{1}(k) f^{2}(k)+\lambda_{2}(k) g^{2}(k)+2 \lambda_{3}(k) f(k) g(k)\right|^{2} \lambda^{-2}(k) \sin ^{2}\left[2 \lambda(k)\left(s-s_{0}\right)\right] \mathrm{d} \mu(k)<\infty,
$$

and the strong continuity condition is

$$
\begin{aligned}
0=\lim _{s \rightarrow s_{0}} \int_{\tilde{\mathbb{X}}} \mid\left\{\left[\lambda_{1}(k)|f(k)|^{2}+\lambda_{2}(k)|g(k)|^{2}\right.\right. & \left.+\lambda_{3}(k)(f(k) \bar{g}(k)+\bar{f}(k) g(k))\right] \sin \left[2 \lambda(k)\left(s-s_{0}\right)\right] \\
& \left.+i \lambda(k)\left(\cos \left[2 \lambda(k)\left(s-s_{0}\right)\right]-1\right)\right\} a_{k}\left(s_{0}\right) \\
+ & {\left.\left[\lambda_{1}(k) \bar{f}^{2}(k)+\lambda_{2}(k) \bar{g}^{2}(k)+2 \lambda_{3}(k) \bar{f}(k) \bar{g}(k)\right] \sin \left[2 \lambda(k)\left(s-s_{0}\right)\right] \bar{a}_{k}\left(s_{0}\right)\right|^{2} \lambda^{-2}(k) \mathrm{d} \mu(k) . }
\end{aligned}
$$

\section{B. The Gowdy and Schmidt Models}

As we have previously shown in section II, the reduced Hamiltonian (16) for the Gowdy and Schmidt models is

$$
H(t)=\int_{\mathbb{X}}\left(\frac{p_{\phi}^{2}(x)}{4 t}+t \phi^{\prime 2}(x)\right) \mathrm{d} x .
$$

After redefining the time parameter as $t=e^{T}$ and using the cosine transform (19) we get

$$
H(T)=\frac{1}{2} \int_{\tilde{\mathbb{X}}}\left(p_{\phi}^{2}(k)+k^{2} e^{2 T} \phi^{2}(k)\right) \mathrm{d} \mu(k) .
$$


In both cases there is a conserved quantity (14) of the form

$$
P=\int_{\tilde{\mathbb{X}}} k p_{\phi}(-k) \phi(k) \mathrm{d} \mu(k) .
$$

For the Gowdy model $P$ is constrained to be zero. This is usually taken into account in the quantum theory as a condition on the physical states that is trivially preserved under the time evolution defined by the Hamiltonian (whenever it is well defined).

We discuss now the unitarity of the time evolution defined by the explicit operator $\hat{U}\left(T, T_{0}\right)$ introduced above. The Hamiltonian (40) belongs to the general class (20) with $\Lambda(T)=0$ and $\omega^{2}(T)=e^{2 T}$. Then (21) gives $\Omega^{2}(k, T)=k^{2} e^{2 T}$ and, in this case, the general solution to the Ermakov-Pinney equation (26) can be written in terms of Bessel functions as

$$
\rho(k, T)=\left[A J_{0}^{2}\left(|k| e^{T}\right)+2 B J_{0}\left(|k| e^{T}\right) Y_{0}\left(|k| e^{T}\right)+C Y_{0}^{2}\left(|k| e^{T}\right)\right]^{1 / 2}
$$

with $A C-B^{2}=\pi^{2} / 4$. It is possible to understand the difficulties to get a unitary evolution operator as the impossibility of fulfilling the unitarity conditions for some of the operators used to build $\hat{U}\left(T, T_{0}\right)$, in particular the condition (36) for $\hat{\mathcal{S}}\left(T, T_{0}\right)$. To show this we look at the leading asymptotic behavior of $\lambda_{3}(k)=(1 / 2) \log \left[\rho\left(k, T_{0}\right) / \rho(k, T)\right]$ when $|k| \rightarrow \infty$ that is given by $\left(T-T_{0}\right) / 4$ plus a bounded periodic function of $|k|$. We have also $\lambda_{1}(k) / \lambda_{3}(k)=$ $-2\left[\dot{\rho}\left(k, T_{0}\right) / \rho\left(k, T_{0}\right)-\Lambda\left(T_{0}\right)\right] \sim 1$. This shows that the convergence of (36) would require $\lim _{|k| \rightarrow \infty} f(k) / g(k)=-2$ which is not compatible with $(28)$ and, hence, $\hat{\mathcal{S}}\left(T, T_{0}\right)$ cannot be unitary.

The way this factor fails to be unitary suggests that one could avoid this problem by introducing from the start (say at the Lagrangian level and, hence, before obtaining the Hamiltonian) a new field $\xi$ differing from $\phi$ in a certain time dependent factor. The reason is to modify the leading asymptotics of $\lambda_{3}(k)$ to improve the convergence of (36). A possible way to change that behavior within the conceptual scheme that we are using here is to factorize the scalar field $\phi(k, T)=h(T) \xi(k, T)$ for an appropriately chosen function $h(T)$. It is always possible to find a unique (modulo a multiplicative constant) time redefinition for a given $h$ in such a way that the new field $\xi$ satisfies, again, a differential equation for a harmonic oscillator with time (and $k$ ) dependent frequency. It could be possible, in principle, to obtain general conditions for $h$ that guarantee that the function $\lambda_{3}(k)$ has the right asymptotic behavior in $k$. Physically one would expect that a choice for which the frequency squared is a sum of $k^{2}$ plus a decreasing function of time would work as the system would approach a free one for which the evolution operator $\hat{U}$ in the form written above is well defined and unitary. The essentially unique way to do this (see appendix C) is to introduce a new field $\xi$ satisfying

$$
\xi(x, t)=\sqrt{t} \phi(x, t)
$$

as was done in $[14,19]$. Here the appropriate time variable is precisely the original one $t=e^{T}$. The Hamiltonian in terms of $\xi(x)$ and its canonically conjugate momentum $p_{\xi}(x)$ becomes [14]

$$
H(t)=\int_{\mathbb{X}}\left(\frac{p_{\xi}^{2}(x)}{4}+\frac{\xi(x) p_{\xi}(x)}{2 t}+\xi^{\prime 2}(x)\right) \mathrm{d} x .
$$

Notice that this is not the Hamiltonian considered in [19]. Although it is related to it by a canonical transformation, the two quantum dynamics are different because the same wave function at an initial time $t_{0}$ evolves differently. Diagonalizing (42) by using the transform (19), defined now for $\xi$ and $p_{\xi}$, and introducing the corresponding operators $\hat{\xi}(k)$ and $\hat{p}_{\xi}(k)$ we get

$$
\hat{H}(t)=\frac{1}{2} \int_{\tilde{\mathbb{X}}}\left(\hat{p}_{\xi}^{2}(k)+\frac{1}{2 t}\left[\hat{p}_{\xi}(k) \hat{\xi}(k)+\hat{\xi}(k) \hat{p}_{\xi}(k)\right]+k^{2} \hat{\xi}^{2}(k)\right) \mathrm{d} \mu(k) .
$$

As we can see the Hamiltonian (42) belongs to the class (18) with $\Lambda(t)=1 /(2 t), \omega^{2}(t)=1$. In this case, by using (21), $\Omega^{2}(k, t)=k^{2}+1 /\left(4 t^{2}\right)$ and a solution to the corresponding Ermakov-Pinney equation (26) is

$$
\rho(k, t)=\sqrt{\pi t / 2}\left[J_{0}^{2}(|k| t)+Y_{0}^{2}(|k| t)\right]^{1 / 2} .
$$

The asymptotic behavior of the functions associated to this solution that appear in the unitarity conditions $(34,36,38)$ is given in the following table 


\begin{tabular}{|c|l|l|}
\hline \hline Function & \multicolumn{1}{|c|}{$|k| \rightarrow \infty$} & \multicolumn{1}{|c|}{$|k| \rightarrow 0$} \\
\hline \hline$\rho(k, t)$ & $\sim \frac{1}{\sqrt{|k|}}$ & $\sim \sqrt{\frac{2 t}{\pi}} \log (|k| t)$ \\
\hline $\int_{t_{0}}^{t} \frac{d \tau}{\rho^{2}(k, \tau)}$ & $\sim C\left(t, t_{0}\right)|k|$ & $\sim \frac{\log \left(t / t_{0}\right)}{\log ^{2}|k|}$ \\
\hline $\log \frac{\rho\left(k, t_{0}\right)}{\rho(k, t)}$ & $\sim \frac{t_{0}^{2}-t^{2}}{16|k|^{2} t^{2} t_{0}^{2}}$ & $\sim \log \left(t_{0} / t\right)\left[\frac{1}{2}+\frac{1}{\log |k|}\right]$ \\
\hline$\frac{\dot{\rho}(k, t)}{\rho(k, t)}-\Lambda(t)$ & $\sim \frac{1}{8|k|^{2} t^{3}}-\frac{1}{2 t}$ & $\sim \frac{1}{t \log (|k| t)}$ \\
\hline \hline
\end{tabular}

where we do not need the specific form of $C\left(t, t_{0}\right)$. They can be easily derived from (43) with the exception of the second one that requires the use of equation (B2) of appendix B. Choosing, for example,

$$
f(k)=1 / \sqrt{2(1+|k|)} \text { and } g(k)=-i \sqrt{(1+|k|) / 2}
$$

it is straightforward to check that the unitarity conditions $(34,36,38)$ are satisfied together with $(28)$. Finally, strong continuity in the auxiliary parameter $s$ follows from $(35,37,39)$, by using the asymptotic expansions given above and the fact that $a_{k}\left(s_{0}\right)$ is square summable. We conclude, hence, that the exponents (times $i$ ) in $\hat{\mathcal{D}}\left(t, t_{0}\right), \hat{\mathcal{S}}\left(t, t_{0}\right)$, and $\hat{\mathcal{R}}\left(t, t_{0}\right)$ are self-adjoint and these are unitary.

In order to verify that $\hat{U}\left(t, t_{0}\right)$ satisfies the evolution equation one must be able to compute derivatives in $t$. This can be shown to be formally possible due to the fact that $t$ derivatives of the exponent trivially commute with it. Also, one can see (appendix A) that the evolution of the field and momentum operators generated by $\hat{U}\left(t, t_{0}\right)$ is differentiable and their time derivatives are obtained as commutators with the Hamiltonian. This allows us to make sense of the time derivative of $\hat{U}\left(t, t_{0}\right)$ indirectly through its action on the basic operators. Of course, one can try to study the behavior in $\left(t, t_{0}\right)$ in a rigorous mathematical sense from first principles but this is beyond the scope of the present paper.

\section{CONCLUSIONS AND COMMENTS}

Some comments are in order now. First of all it must be said that if one is only interested in Fock space representations the evolution of $n$-particle states can be simply derived from the evolution of creation operators in the Heisenberg picture (that can be directly read from the classical dynamics of the system) and the evolution of the vacuum state. Furthermore, the vacuum evolution can be written in closed form as in [32,33]. This provides the matrix elements of the evolution operator in a concrete Hilbert space basis. A potential advantage of the framework discussed in this paper is that one can, in principle, try to use non-Fock representations to define the evolution operator given in closed form by $(23,24,25)$. A second comment is that

$$
\frac{\hat{\xi}(x)}{\sqrt{t}}=\frac{1}{\sqrt{2 \pi t}} \int_{\tilde{\mathbb{X}}}\left[f(k) \hat{a}_{k}+\bar{f}(k) \hat{a}_{k}^{\dagger}\right] \cos \left(k x-\frac{\pi}{4}\right) \mathrm{d} \mu(k),
$$

interpreted as an explicitly time dependent operator in the Schrödinger picture evolves, in the Heisenberg picture, as the classical scalar field $\phi(x, t)$ that encodes the physical degrees of freedom of the Gowdy and Schmidt models. This evolution is perfectly well defined and unitary. As we can see the problem is not that it is impossible to define a unitary evolution for an object that behaves as the scalar field $\phi$ but rather the impossibility of doing this with a representation of the type

$$
\hat{\phi}(k)=f(k) \hat{a}_{k}+\bar{f}(k) \hat{a}_{k}^{\dagger}, \quad \hat{p}_{\phi}(k)=g(k) \hat{a}_{k}+\bar{g}(k) \hat{a}_{k}^{\dagger} .
$$

We want to emphasize that the use of the field $\xi$ defined in (41) is as justified as that of $\phi$ because it can be chosen as the fundamental object already at the Lagrangian level. It is important, however, to realize that the quantum dynamics must then be obtained from the corresponding Hamiltonian. In this respect it should be noticed that it is possible to write down the Hamiltonian corresponding to the dynamics considered in [19], build the unitary evolution operator in that case with our methods.

The possible uses of the approach that we have presented here are manifold. In addition to the discussion of consistency issues related to the unitarity of the time evolution of the system other problems that may be tackled 
are the generalization of the coherent and squeezed states for the harmonic oscillator with time dependent frequency to these quantum cosmological models, the study of particle creation, and the introduction and discussion of new representations to quantize the system.

\section{Acknowledgments}

The authors want to thank J. Cortez, I. Garay, L. Garay, J. M. Martín García, G. Mena Marugán, and M. Varadarajan for their insightful comments. This work was supported in part by the Spanish MEC grant BFM200204031-C02-02 and D. Gómez Vergel acknowledges the financial support provided by CSIC through the Introduction to Research assistantship and I3P programs.

\section{APPENDIX A: COMPUTING THE UNITARY EVOLUTION OPERATOR}

We give here some details about the construction of the formal evolution operators for the kind of system considered in the paper. In order to quantize the model defined by the Hamiltonian (20), we promote the field and momentum to formal operators $\hat{\varphi}(k), \hat{\pi}(k)$, satisfying the canonical commutation relations $[\hat{\varphi}(k), \hat{\pi}(q)]=i \hbar \delta(k, q)$, and symmetrize the cross term in the classical Hamiltonian to arrive at (22). We want to solve now the Schrödinger equation ${ }^{5}$ $i \hbar \partial_{t}|\psi(t)\rangle=\hat{H}(t)|\psi(t)\rangle$. The strategy that we follow is to generalize the results already known for a single harmonic oscillator with a time dependent frequency to an infinite system of uncoupled harmonic oscillators [26, 27]. In order to obtain the quantum evolution operator, we define

$$
\begin{aligned}
& \hat{D}(t ; f):=\exp \left(-\frac{i}{2 \hbar} \int_{\tilde{\mathbb{X}}} f(k, t) \hat{\varphi}^{2}(k) \mathrm{d} \mu(k)\right) \\
& \hat{S}(t ; g):=\exp \left(\frac{i}{2 \hbar} \int_{\tilde{\mathbb{X}}} g(k, t)[\hat{\varphi}(k) \hat{\pi}(k)+\hat{\pi}(k) \hat{\varphi}(k)] \mathrm{d} \mu(k)\right)
\end{aligned}
$$

depending on some functions $f$ and $g$ that will be fixed in the following. Let us consider their product $\hat{T}(t ; f, g)=$ $\hat{S}(t ; g) \hat{D}(t ; f)$ and introduce the state vector $|\chi(t ; f, g)\rangle=\hat{T}(t ; f, g)|\psi(t)\rangle$ written in terms of a solution to the Schrödinger equation $|\psi(t)\rangle$. We find now the equation that $|\chi(t ; f, g)\rangle$ must satisfy and choose the functions $f(k, t)$ and $g(k, t)$ in such a way that the evolution for this vector can be easily obtained

$$
\begin{aligned}
i \hbar \partial_{t}|\chi(t ; f, g)\rangle= & \left(\hat{T}(t ; f, g) \hat{H}(t) \hat{T}^{\dagger}(t ; f, g)-i \hbar \hat{T}(t ; f, g) \partial_{t} \hat{T}^{\dagger}(t ; f, g)\right)|\chi(t ; f, g)\rangle \\
= & \frac{1}{2} \int_{\tilde{\mathbb{X}}} \mathrm{d} \mu(k)\left(\mathrm{e}^{-2 g(k, t)} \hat{\pi}^{2}(k)+[f(k, t)+\Lambda(t)-\dot{g}(k, t)][\hat{\varphi}(k) \hat{\pi}(k)+\hat{\pi}(k) \hat{\varphi}(k)]\right. \\
& \left.+\mathrm{e}^{2 g(k, t)}\left[f^{2}(k, t)+k^{2} \omega^{2}(t)+2 \Lambda(t) f(k, t)+\dot{f}(k, t)\right] \hat{\varphi}^{2}(k)\right)|\chi(t ; f, g)\rangle
\end{aligned}
$$

where the dot denotes $\partial_{t}$ and we have used the following relations satisfied by $\hat{D}(t ; f)$ and $\hat{S}(t ; g)$

$$
\begin{aligned}
& \hat{D}(t ; f) \hat{\varphi}(k) \hat{D}^{\dagger}(t ; f)=\hat{\varphi}(k), \\
& \hat{D}(t ; f) \hat{\pi}(k) \hat{D}^{\dagger}(t ; f)=\hat{\pi}(k)+f(k, t) \hat{\varphi}(k), \\
& \hat{S}(t ; g) \hat{\varphi}(k) \hat{S}^{\dagger}(t ; g)=\hat{\varphi}(k) \mathrm{e}^{g(k, t)} \\
& \hat{S}(t ; g) \hat{\pi}(k) \hat{S}^{\dagger}(t ; g)=\hat{\pi}(k) \mathrm{e}^{-g(k, t)} .
\end{aligned}
$$

The form of (A1) suggests to choose functions $f, g$ such that

$$
\begin{aligned}
& f(k, t)+\Lambda(t)=\dot{g}(k, t) \\
& f^{2}(k, t)+k^{2} \omega^{2}(t)+2 \Lambda(t) f(k, t)+\dot{f}(k, t)=\mathrm{e}^{-4 g(k, t)} .
\end{aligned}
$$

${ }^{5}$ We assume that a suitable Hilbert space representation exists. 
The equation for $|\chi(t)\rangle$ simplifies then to

$$
i \hbar \partial_{t}|\chi(t)\rangle=\frac{1}{2} \int_{\tilde{\mathbb{X}}} \mathrm{d} \mu(k) \mathrm{e}^{-2 g(k, t)}\left[\hat{\pi}^{2}(k)+\hat{\varphi}^{2}(k)\right]|\chi(t)\rangle .
$$

A concrete way to achieve this is to take $g(k, t)=\log \rho(k, t)$ [and, hence, $f(k, t)=\dot{\rho}(k, t) / \rho(k, t)-\Lambda(t)$ ] with the new function $\rho(k, t)$ obeying the auxiliary Ermakov-Pinney equation

$$
\ddot{\rho}(k, t)+\Omega^{2}(k, t) \rho(k, t)=\rho^{-3}(k, t) .
$$

Here the dot denotes $\partial_{t}$ and $\Omega^{2}(k, t)=k^{2} \omega^{2}(t)-\Lambda^{2}(t)-\dot{\Lambda}(t)$, that we will suppose to be positive in the following. As we can see this equation is closely related to the classical equation of motion for a harmonic oscillator with timedependent frequency $\Omega(k, t)$. In terms of this new function the state vector $|\chi(t)\rangle$ satisfies the differential equation

$$
i \hbar \partial_{t}|\chi(t)\rangle=\frac{1}{2}\left(\int_{\tilde{\mathbb{X}}} \frac{1}{\rho^{2}(k, t)}\left[\hat{\pi}^{2}(k)+\hat{\varphi}^{2}(k)\right] \mathrm{d} \mu(k)\right)|\chi(t)\rangle
$$

that can be easily solved to get

$$
|\chi(t)\rangle:=\hat{R}\left(t, t_{0}\right)\left|\chi\left(t_{0}\right)\right\rangle=\exp \left(-\frac{i}{2 \hbar} \int_{\tilde{\mathbb{X}}}\left(\int_{t_{0}}^{t} \frac{\mathrm{d} \tau}{\rho^{2}(k, \tau)}\right)\left[\hat{\pi}^{2}(k)+\hat{\varphi}^{2}(k)\right] \mathrm{d} \mu(k)\right)\left|\chi\left(t_{0}\right)\right\rangle .
$$

At this point one can build the solution to the original Schrödinger equation from $|\chi(t)\rangle$ and the operator $\hat{T}(t)$.

The solutions to (A2) have some interesting features, in particular they never vanish and are such that $|\chi(t)\rangle$ is independent of the initial conditions chosen for $\rho(k, t)$, as will be shown in appendix B. Other choices for the function $g(k, t)$ allow the formal solution of the equation for $|\chi(t)\rangle$ but are problematic because the evolution operators thus obtained are not well defined for all values of $t[27]$. Finally, going back to the original state vector $|\psi(t)\rangle$, we obtain the formal quantum evolution operator ${ }^{6}$

$$
\hat{U}\left(t, t_{0}\right)=\hat{T}^{\dagger}(t) \hat{R}\left(t, t_{0}\right) \hat{T}\left(t_{0}\right)
$$

with $\hat{T}(t)=\hat{S}(t) \hat{D}(t)$ and

$$
\begin{aligned}
& \hat{D}(t)=\exp \left(-\frac{i}{2 \hbar} \int_{\tilde{\mathbb{X}}}\left[\frac{\dot{\rho}(k, t)}{\rho(k, t)}-\Lambda(t)\right] \hat{\varphi}^{2}(k) \mathrm{d} \mu(k)\right), \\
& \hat{S}(t)=\exp \left(\frac{i}{2 \hbar} \int_{\tilde{\mathbb{X}}} \log \rho(k, t)[\hat{\varphi}(k) \hat{\pi}(k)+\hat{\pi}(k) \hat{\varphi}(k)] \mathrm{d} \mu(k)\right), \\
& \hat{R}\left(t, t_{0}\right)=\exp \left(-\frac{i}{2 \hbar} \int_{\tilde{\mathbb{X}}}\left(\int_{t_{0}}^{t} \frac{\mathrm{d} \tau}{\rho^{2}(k, \tau)}\right)\left[\hat{\pi}^{2}(k)+\hat{\varphi}^{2}(k)\right] \mathrm{d} \mu(k)\right) .
\end{aligned}
$$

It is clear, by construction, that if we can find a suitable representation for $\hat{\varphi}(k)$ and $\hat{\pi}(k)$ as self-adjoint operators on a certain Hilbert space, $\hat{U}\left(t, t_{0}\right)$ satisfies

$$
\begin{aligned}
& \hat{U}\left(t_{0}, t_{0}\right)=\mathbb{I}, \\
& \hat{U}\left(t, t_{0}\right)^{-1}=\hat{U}\left(t, t_{0}\right)^{\dagger}, \\
& \hat{U}\left(t_{2}, t_{0}\right)=\hat{U}\left(t_{2}, t_{1}\right) \hat{U}\left(t_{1}, t_{0}\right), \\
& i \hbar \partial_{t} \hat{U}\left(t, t_{0}\right)=\hat{H}(t) \hat{U}\left(t, t_{0}\right)
\end{aligned}
$$

where $\hat{H}(t)$ is the quantum Hamiltonian (22). It is interesting to notice (see appendix B) that

$$
\sin \left(\int_{t_{0}}^{t} \frac{\mathrm{d} \tau}{\rho^{2}(k, \tau)}\right)=\frac{u(k, t)}{\rho(k, t) \rho\left(k, t_{0}\right)}
$$

\footnotetext{
${ }^{6}$ The evolution of a state vector from time $t_{0}$ to $t$ is given by $|\psi(t)\rangle=\hat{U}\left(t, t_{0}\right)\left|\psi\left(t_{0}\right)\right\rangle$.
} 
where $u(k, t)$ is the unique solution to the homogeneous equation $\ddot{u}(k, t)+\Omega^{2}(k, t) u(k, t)=0$ satisfying $u\left(k, t_{0}\right)=0$ and $\dot{u}\left(k, t_{0}\right)=1$. This allows us to write explicit and closed expressions for $\hat{U}\left(t, t_{0}\right)$ whenever we can give closed solutions to the previous equation for $u(k, t)$ because solutions to the Ermakov equation can be obtained from these.

In order to discuss the conditions that the representations for the field and momentum must satisfy it is convenient to factorize it as $\hat{U}\left(t, t_{0}\right)=\hat{\mathcal{D}}\left(t, t_{0}\right) \hat{\mathcal{S}}\left(t, t_{0}\right) \hat{\mathcal{R}}\left(t, t_{0}\right)$ with

$$
\begin{aligned}
& \hat{\mathcal{D}}\left(t, t_{0}\right):=\hat{D}^{\dagger}(t) \hat{D}\left(t_{0}\right), \\
& \hat{\mathcal{S}}\left(t, t_{0}\right):=\hat{D}^{\dagger}\left(t_{0}\right) \hat{S}^{\dagger}(t) \hat{S}\left(t_{0}\right) \hat{D}\left(t_{0}\right), \\
& \hat{\mathcal{R}}\left(t, t_{0}\right):=\hat{D}^{\dagger}\left(t_{0}\right) \hat{S}^{\dagger}\left(t_{0}\right) \hat{R}\left(t, t_{0}\right) \hat{S}\left(t_{0}\right) \hat{D}\left(t_{0}\right) .
\end{aligned}
$$

These expressions directly lead to $(23,24,25)$. The evolution of the field is

$$
\begin{aligned}
\hat{\varphi}_{H}\left(k, t_{0}, t\right) & =\rho(k, t)\left[\frac{1}{\rho\left(k, t_{0}\right)} \cos \left(\int_{t_{0}}^{t} \frac{\mathrm{d} \tau}{\rho^{2}(k, \tau)}\right)-\rho\left(k, t_{0}\right)\left(\frac{\dot{\rho}\left(k, t_{0}\right)}{\rho\left(k, t_{0}\right)}-\Lambda\left(t_{0}\right)\right) \sin \left(\int_{t_{0}}^{t} \frac{\mathrm{d} \tau}{\rho^{2}(k, \tau)}\right)\right] \hat{\varphi}(k) \\
& +\rho(k, t) \rho\left(k, t_{0}\right) \sin \left(\int_{t_{0}}^{t} \frac{\mathrm{d} \tau}{\rho^{2}(k, \tau)}\right) \hat{\pi}(k) .
\end{aligned}
$$

Similarly we can compute the momentum operator in the Heisenberg picture

$$
\frac{\mathrm{d} \hat{\varphi}_{H}}{\mathrm{~d} t}\left(k, t_{0}, t\right)=-\frac{i}{\hbar}\left[\hat{\varphi}_{H}\left(k, t_{0}, t\right), \hat{H}_{H}(t)\right]=\hat{\pi}_{H}\left(k, t_{0}, t\right) .
$$

It is straightforward to show that these are formal solutions to the classic equations of motion -as one would expect given the quadratic nature of the Hamiltonian- because the coefficients of $\hat{\varphi}(k)$ and $\hat{\pi}(k)$ are solutions to the classical equations $\ddot{\varphi}(k, t)+\Omega^{2}(k, t) \varphi(k, t)=0$.

\section{APPENDIX B: SOME PROPERTIES OF THE ERMAKOV-PINNEY EQUATION}

We discuss in this appendix some properties of the Ermakov-Pinney equation

$$
\ddot{\rho}(t)+\Omega^{2}(t) \rho(t)=\rho^{-3}(t)
$$

that are relevant to show the independence of the evolution operators from the choice of initial conditions. The most general analytic solution to this equation ${ }^{7}$ can be written as

$$
\rho(t)=+\left[A u^{2}(t)+2 B u(t) v(t)+C v^{2}(t)\right]^{1 / 2}
$$

where $u(t)$ and $v(t)$ are linearly independent solutions to the time-dependent harmonic oscillator equation $\ddot{\varphi}(t)+$ $\Omega^{2}(t) \varphi(t)=0, A, B, C$ are constants satisfying $A C-B^{2}=1 / W^{2}[u, v]$, and $W[u, v]:=u \dot{v}-\dot{u} v$ denotes the (time independent) Wronskian of the pair of solutions $u$ and $v$. The square of a solution can be expressed as

$$
\rho^{2}(t)=[u(t) v(t)]\left[\begin{array}{ll}
A & B \\
B & C
\end{array}\right]\left[\begin{array}{l}
u(t) \\
v(t)
\end{array}\right]
$$

where $(u, v) \neq(0,0)$ for all $t$ (zeros of solutions $u(t)$ and $v(t)$ alternate and never coincide as a consequence of Sturm's theorem). Hence

$$
\left|\begin{array}{ll}
A & B \\
B & C
\end{array}\right|=A C-B^{2}=W^{-2}>0,
$$

therefore, the quadratic form $\rho^{2}(t)$ is positive definite and $\rho(t)>0$ for all $t$.

\footnotetext{
${ }^{7}$ Here we drop the parameter $k$ that appears in (A2) because the results are trivially generalized to that case.
} 
The general solution of the time-dependent harmonic oscillator equation $\ddot{\varphi}(t)+\Omega^{2}(t) \varphi(t)=0$ can be expressed as

$$
\varphi(t)=\left[\frac{\rho(t)}{\rho_{0}} \cos \left(\int_{t_{0}}^{t} \frac{\mathrm{d} \tau}{\rho^{2}(\tau)}\right)-\rho(t) \dot{\rho}_{0} \sin \left(\int_{t_{0}}^{t} \frac{\mathrm{d} \tau}{\rho^{2}(\tau)}\right)\right] \varphi_{0}+\rho(t) \rho_{0} \sin \left(\int_{t_{0}}^{t} \frac{\mathrm{d} \tau}{\rho^{2}(\tau)}\right) \dot{\varphi}_{0}
$$

where $\rho(t)$ is an arbitrary solution to the associated Ermakov-Pinney equation $\ddot{\rho}(t)+\Omega^{2}(t) \rho(t)=\rho^{-3}(t)$; the subindex 0 denotes evaluation at $t_{0}$. Notice that the positivity of $\rho(t)$ guarantees that the previous expression is well-defined for any value of $t$. As $\varphi(t)$ is uniquely determined by the initial conditions $\varphi_{0}$ and $\dot{\varphi}_{0}$ the functions

$$
\frac{\rho(t)}{\rho_{0}} \cos \left(\int_{t_{0}}^{t} \frac{\mathrm{d} \tau}{\rho^{2}(\tau)}\right)-\rho(t) \dot{\rho}_{0} \sin \left(\int_{t_{0}}^{t} \frac{\mathrm{d} \tau}{\rho^{2}(\tau)}\right) \quad \text { and } \quad \rho(t) \rho_{0} \sin \left(\int_{t_{0}}^{t} \frac{\mathrm{d} \tau}{\rho^{2}(\tau)}\right)
$$

must be independent of the choice of the solution $\rho(t)$ to the Ermakov equation. This result has some interesting consequences regarding the quantum evolution operators studied in the paper. First of all it is possible to show that the unitary evolution operator for a single quantum harmonic oscillator with time-dependent frequency (obtained with the techniques explained in the paper) is independent of the choice of $\rho(t)$. This is obvious if one looks at the evolution for the field operator given by (A3). Second, it is easy to see from (B1) that the solution $u(t)$ to the equation $\ddot{u}(t)+\Omega^{2}(t) u(t)=0$ satisfying $u_{0}=0$ and $\dot{u}_{0}=1$ is

$$
u(t)=\rho(t) \rho_{0} \sin \left(\int_{t_{0}}^{t} \frac{\mathrm{d} \tau}{\rho^{2}(\tau)}\right)
$$

and, hence,

$$
\sin \left(\int_{t_{0}}^{t} \frac{\mathrm{d} \tau}{\rho^{2}(\tau)}\right)=\frac{u(t)}{\rho(t) \rho_{0}}
$$

As the integrand in the previous expression is positive, the integral is a strictly increasing function of $t$. The value of the integral itself is given in this case by the unique continuous and increasing extension to $\mathbb{R}$ of the arcsin function with argument $u(t) /\left[\rho(t) \rho_{0}\right]$. This is used to obtain one of the asymptotic expansions appearing in IV B.

\section{APPENDIX C: ON THE DEFINITION OF THE FIELD $\xi$}

We will discuss in this appendix the obtention of the field $\xi$ introduced in section IV B. The reduced phase space for the Gowdy and Schmidt models can be coordinatized by $\left(\phi(k), p_{\phi}(k)\right)$ and the Hamilton equations derived from (40) lead to the time-dependent harmonic oscillator equation

$$
\partial_{T}^{2} \phi(k, T)+k^{2} e^{2 T} \phi(k, T)=0 .
$$

It is always possible to write (C1) in terms of a new field $\xi$ defined by

$$
\phi(k, T)=h(T) \xi(k, T), \quad \text { with } \quad h>0,
$$

to give

$$
\partial_{T}^{2} \xi(k, T)+2 \frac{h^{\prime}(T)}{h(T)} \partial_{T} \xi(k, T)+\left(\frac{h^{\prime \prime}(T)}{h(T)}+k^{2} e^{2 T}\right) \xi(k, T)=0 ;
$$

here $h^{\prime}$ and $h^{\prime \prime}$ denote the first and second derivative of the function $h$ respectively. After performing a time redefinition $t(T)$, with $t^{\prime}(T)>0$, the equation (C2) can be written as

$$
\partial_{t}^{2} \xi(k, t)+t^{\prime-2}(T)\left(t^{\prime \prime}(T)+2 \frac{t^{\prime}(T) h^{\prime}(T)}{h(T)}\right) \partial_{t} \xi(k, t)+t^{\prime-2}(T)\left(\frac{h^{\prime \prime}(T)}{h(T)}+k^{2} e^{2 T}\right) \xi(k, t)=0 .
$$

Although (C3) is not yet a time-dependent harmonic oscillator equation, it is always possible to eliminate the $\left(\partial_{t} \xi\right)$ term by imposing

$$
t^{\prime \prime}+2 \frac{t^{\prime} h^{\prime}}{h}=0
$$


The general solution of $(\mathrm{C} 4)$ is

$$
t(T)=t_{0} \int_{T_{0}}^{T} \frac{\mathrm{d} \tau}{h^{2}(\tau)}
$$

where $t_{0}>0$ and $T_{0}$ are constants of integration. With these assumptions the equation (C3) becomes

$$
\partial_{t}^{2} \xi(k, t)+\left(\frac{h^{\prime \prime}(T)}{t^{\prime 2}(T) h(T)}+k^{2} \frac{e^{2 T}}{t^{\prime 2}(T)}\right) \xi(k, t)=0
$$

with $t(T)$ given by (C5). This last equation is now of the form $\partial_{t}^{2} \xi(k, t)+\Omega^{2}(k, t) \xi(k, t)=0$, with

$$
\Omega^{2}(k, t)=\frac{h^{\prime \prime}(T)}{t^{\prime 2}(T) h(T)}+k^{2} \frac{e^{2 T}}{t^{\prime 2}(T)},
$$

for any choice of $h$. Finally, it is possible to fix the function $h$ by requiring that $\Omega^{2}$ is a sum of $k^{2}$ plus a function of time. Explicitly, by demanding $e^{2 T} / t^{\prime 2}(T)=1$, we get

$$
t(T)=e^{T}-e^{T_{0}}, \quad h(T)=\sqrt{t_{0}} e^{-T / 2}
$$

or, equivalently,

$$
T=\log \left(t+e^{T_{0}}\right), \quad h=\frac{\sqrt{t_{0}}}{\sqrt{t+e^{T_{0}}}} .
$$

Particularizing (C6) for $t_{0}=1$ and $T_{0} \rightarrow-\infty$ we finally arrive at (41)

$$
\xi(k, t)=\sqrt{t} \phi(k, t)
$$

where the field $\xi$ satisfies

$$
\partial_{t}^{2} \xi(k, t)+\left(\frac{1}{4 t^{2}}+k^{2}\right) \xi(k, t)=0
$$

[1] A. Einstein and N. Rosen, J. Franklin Inst. 223, 43 (1937).

[2] B. G. Schmidt, Class. Quantum Grav. 13, 2811 (1996).

[3] R. H. Gowdy, Phys. Rev. Lett. 27, 826 (1971).

[4] R. H. Gowdy, Ann. Phys. 83, 203 (1974).

[5] A. Ashtekar and M. Pierri, J. Math. Phys. 37, 6250 (1996), gr-qc/9606085.

[6] M. Pierri, Int. J. Mod. Phys. D11, 135 (2002), gr-qc/0101013.

[7] C. Beetle, Adv. Theor. Math. Phys. 2, 471 (1998), gr-qc/9801107.

[8] K. Kuchař, Phys. Rev. D4, 955 (1971).

[9] A. Ashtekar and M. Varadarajan, Phys. Rev. D50, 4944 (1994), gr-qc/9406040.

[10] A. Ashtekar, Phys. Rev. Lett. 77, 4864 (1996), gr-qc/9610008.

[11] J. F. Barbero G., I. Garay, and E. J. S. Villaseñor, Phys. Rev. Lett. 95, 050501 (2005), gr-qc/0506093.

[12] J. F. Barbero G., G. A. Mena Marugán, and E. J. S. Villaseñor, Phys. Rev. D67, 124006 (2003), gr-qc/0304047.

[13] C. W. Misner, Phys. Rev. D 8, 3271 (1973).

[14] B. K. Berger, Ann. Phys. 83, 458 (1974).

[15] B. K. Berger, Phys. Rev. D11, 2770 (1975).

[16] R. M. Wald, Quantum field theory in curved space-time and black hole thermodynamics (The University of Chicago Press, Chicago and London, 1994).

[17] C. G. Torre, Phys. Rev. D66, 084017 (2002), gr-qc/0206083.

[18] A. Corichi, J. Cortez, and H. Quevedo, Int. J. Mod. Phys. D11, 1451 (2002), gr-qc/0204053.

[19] A. Corichi, J. Cortez, and G. A. Mena Marugán, Phys. Rev. D73, 041502(R) (2006), gr-qc/0510109.

[20] A. Corichi, J. Cortez, and G. A. Mena Marugán, Phys. Rev. D73, 084020 (2006), gr-qc/0603006.

[21] V. Husain, Class. Quant. Grav. 4, 1587 (1987).

[22] P. Chrusciel, Annals Phys. 202, 100 (1990).

[23] B. K. Berger, P. T. Chrusciel, and V. Moncrief, Ann. Phys. 237, 322 (1995), gr-qc/9404005.

[24] R. Geroch, J. Math. Phys. 12, 918 (1971). 
[25] A. O. Barvinsky, Phys. Rept. 230, 237 (1993).

[26] H. R. Lewis Jr. and W. B. Riesenfeld, J. Math. Phys. 10 Number 8, 1458 (1969).

[27] M. Fernández Guasti and H. Moya-Cessa, J. Phys. A 36, 2069 (2003).

[28] C. Bertoni, F. Finelli, and G. Venturi, Phys. Lett. A237, 331 (1998), gr-qc/9706061.

[29] F. Finelli, A. Gruppuso, and G. Venturi, Class. Quant. Grav. 16, 3923 (1999), gr-qc/9909007.

[30] F. Finelli, G. P. Vacca, and G. Venturi, Phys. Rev. D58, 103514 (1998), gr-qc/9712098.

[31] R. M. Hawkins and J. E. Lidsey, Phys. Rev. D66, 023523 (2002), astro-ph/0112139.

[32] C. G. Torre and M. Varadarajan, Phys. Rev. D58, 064007 (1998), hep-th/9707221.

[33] K. Pilch and N. P. Warner, Class. Quant. Grav. 4, 1183 (1987). 